\title{
Effects of Different Planting Methods on the Diversity of Endophytic Bacteria in Tobacco
}

\author{
Song Jin ${ }^{1,}$, Xi Gao ${ }^{2}$ Zebin Chen ${ }^{1}$, Yuan Su ${ }^{3}$ Jiani Liư ${ }^{1}$, Lei Yu ${ }^{4}$, Dingkang Wang ${ }^{1}$, \\ Shengguang $\mathrm{Xu}^{5, \mathrm{~b}}$, * \\ ${ }^{1}$ College of Agronomy, Kunming University, Kunming, Yunnan Province, China. \\ ${ }^{2}$ College of Plant Protection, Yunnan Agricultural University, Kunming, Yunnan Province, China. \\ ${ }^{3}$ Key Laboratory of Characteristic Biological Resource Development and Utilization of Colleges and \\ Universities in Yunnan Province, Kunming, Yunnan Province, China. \\ ${ }^{4}$ Yunnan Urban Characteristic Agriculture Engineering Technology Research Center, Kunming, Yunnan \\ Province, China. \\ ${ }^{5}$ Yunnan Institute of Biological Carbon Engineering Research Center, Kunming, Yunnan Province, China. \\ aibmjs@163.com, bsgxu2011@126.com \\ *Corresponding author: Shengguang Xu
}

Keywords: high throughput sequencing; tobacco; planting method; endophytic bacteria; diversity

\begin{abstract}
In this experiment, the tobacco variety Yunyan 87 was cultivated by 3 different planting methods. Afterwards, tobacco leaves were taken as the research material. By using the high throughput sequencing technology, DNA in the 16S rDNA-V4 region of tobacco was sequenced to analyze the abundance and diversity of species, in order to understand the diversities of endophytic bacteria in tobacco under different kinds of planting methods. The results show that the sequence of micro flora richness of 3 tobacco samples was Y6.1 > Y7.1 > Y1.1; the sequence of bacteria diversity was Y7.1 > Y6.1 > Y1.1. The bacterial diversity of Y7.1 was higher than that of Y1.1 and Y6.1. Endophytic bacteria found in samples were distributed in 13 phyla, namely Proteobacteria, Bacteroidetes, Actinobacteria, Acidobacteria, Gemmatimonadetes, Firmicutes, Chloroflexi, Planctomycetes, Nitrospirae, Verrucomicrobia, Thaumarchaeota, Thermomicrobia and Armatimonadetes. Proteobacteria and Actinobacteria were dominant bacteria in Y1.1, which accounted for $61.56 \%$ and $18.26 \%$ of the total bacteria. Proteobacteria, Acidobacteria and Actinobacteria were dominant bacteria in Y6.1, which accounted for $47.42 \%, 19.71 \%$ and $13.40 \%$ of the total bacteria. Proteobacteria, Bacteroidetes and Actinobacteria were dominant bacteria of Y7.1, which accounted for 54.28\%, $15.59 \%$ and $11.24 \%$ of the total bacteria. The distribution of Morganella endophytic bacteria in Y6.1 and Y7.1 was related to the addition of biological carbon. The distribution of Fusobacterium and Proteiniclasticum endophytic bacteria in Y7.1 was related to the inter cropping with marigold.
\end{abstract}

\section{Introduction}

Endophytic bacteria are microorganisms which colonize in healthy plant tissues but do not cause obvious infection symptoms. [1] Endophytic bacteria are closely related to the growth and physiological features of host plants; they produce certain effects on host plants in stress resistance and the formation of metabolites. [2] In addition to the genetic transmission of hosts, endophytic bacteria can also colonize plants through natural holes or wounds on the surface or in rhizosphere of plants; they widely exist in various plants.[3] Hashiba found that the growth of the endophytic Heteroconium chaetospira in cabbage could be inhibited by gardening soil purchased from the market, but the colonization rate of endophytic bacteria was up to $75 \%$ when the plant was cultivated in peat soil containing $0.1 \%$ glucose.[4] Cultivation conditions have great influence on the diversity of endophytic bacteria in plants. [5] Biochar is the solid product transformed from bio organic materials 
(biomass) through high temperature pyrolysis in anoxic or anaerobic environment. Previous studies found that the application of biological carbon could promote the growth and yield of tobacco leaves; the quality and yield of tobacco increased with the increasing amount of biological carbon application; but when the amount of applied biological carbon reached a certain amount $\left(2025 \mathrm{~kg} / \mathrm{hm}^{2}\right)$, the quality of tobacco leaves began to decrease. [6] In order to understand the effect of biological charcoal application and planting pattern changes on the diversity of endophytic bacteria, this study used high throughput sequencing technology to compare the diversity of endophytic bacteria in tobacco plants which were cultivated by 3 planting methods; the aim of this paper was to understand the differences of endophytic bacteria in tobacco under different planting methods, and provide technical reference for the adjustment of tobacco planting modes.

\section{Research Materials and Methods}

\subsection{Soil sample collection}

The tested tobacco variety was Yunyan 87. Three treatment methods were employed. Single cropping tobacco was numbered as Y1.1; single cropping tobacco with $150 \mathrm{~g} /$ strain of biological charcoal application was numbered as Y6.1; tobacco intercropping with marigold and applied with $150 \mathrm{~g} /$ strain of biological charcoal was numbered as Y7.1. The field which had been used in tobacco planting for consecutive three years in Luoshui town of Xuanwei, Yunnan province was selected as the experiment field. When planting tobacco seedlings, biochar and base fertilizers were mixed and applied. On July 18, 2016, mature tobacco leaves without obvious pests or diseases were collected. Samples were immediately stored in aseptic sampling bags and stored at low temperature. Samples were tested within 24 hours.

\subsection{Surface sterilization}

The leaves were washed with aseptic water, then soaked in $75 \%$ (volume fraction) ethanol for 1 minute and washed with sterile water for 3 times. Water in the surface of leaves was dried with sterile filter papers. Effect test on surface sterilization was carried out according to procedure described in reference [7].

\subsection{Extraction of total DNA}

The total DNA of leaves was extracted according to the method of reference [8]. The purity and concentration of DNA were examined by $8 \mathrm{~g} / \mathrm{L}$ agarose gel electrophoresis. Then a proper amount of samples were put into the centrifuge tube and diluted with aseptic water to $1 \mathrm{mg} \cdot \mathrm{L}^{-1}$.

\subsection{PCR amplification, purification and computer sequencing}

Using diluted genomic DNA as the template, 16S-V4 region specific primer with Barcodes was used for PCR. [9] PCR products were detected by agarose gel electrophoresis; the target band products were recovered through gel recovery kit produced by Qiagen Company. Sequencing and bioinformatics analysis were commissioned by Beijing Novogene Corporation.

\section{Research Results and Analysis}

\subsection{Sample diversity analysis}

The dilution curves of OTU of 3 samples tended to flat. As the number of sequences increased, the slope of bacterial dilution curves gradually tended to flat but still increase. It showed that only a small amount of new OTUs were produced when the number of sequencing increased. Information about species quantity would be reflected if the number of sequencing data was large enough.

\subsection{Diversity index of endophytic bacteria}

Through sequencing gene in the V4 region of 16S rRNA, the numbers of original sequences of 3 samples were 60312, 57896 and 60517. After filtering low mass sequences, the numbers of sequences 
were 58938, 56555 and 58403. After removing redundant sequences, the numbers of effective sequences were 58659, 56342 and 58403. Under the similarity level of 97\%, the OTU abundances of endophytic bacteria in 3 tobacco leaves were 1271, 1308 and 1463 respectively. The sequence of numbers was Y1.1 < Y6.1 < Y7.1. The Chao1 index and Shannon index can reflect the abundance and diversity of bacteria respectively. From the diversity index, the sequence of micro flora richness of 3 tobacco samples was Y6.1 > Y7.1 > Y1.1; the sequence of bacteria diversity was Y7.1 > Y6.1 > Y1.1. The diversity of Y7.1 was higher than that of Y1.1 and Y6.1.

\subsection{Correlation analysis on bacterial communities}

1618 OTUs could be found in 3 samples, of which 889 OTUs could be found in all the three samples. 110 OTUs were found in both Y1.1 and Y6.1; 117 OTUs were found in both Y1.1 and Y7.1; 207 OTUs were found in both Y7.1 and Y6.1. The similarity between Y7.1 and Y6.1 were higher in bacterial species. 41 OTUs were found only in Y1.1; 102 OTUs were found only in Y6.1 and 152 OTUs were only found in Y7.1. It could be seen from the test results that the number of OTUs in Y1.1 samples was the least, followed by Y6.1 and Y7.1. With the increasing complexity of planting mode, there was a rising trend in the number of OTUs in 3 samples. The 2 kinds of biological charcoal treated samples had higher bacterial species similarity. The more complex of the planting mode, the more exclusive OTUs would be found. The number of exclusive OTUs in Y7.1 sample was more than that of Y1.1 by 111, and was 3.7 times of the number of Y1.1.

\subsection{The distribution characteristics and abundance of endophytic bacteria}

Endophytic bacteria found in three samples were distributed in 13 phyla, namely Proteobacteria, Bacteroidetes, Actinobacteria, Acidobacteria, Gemmatimonadetes, Firmicutes, Chloroflexi, Planctomycetes, Nitrospirae, Verrucomicrobia, Thaumarchaeota, Thermomicrobia and Armatimonadetes. Proteobacteria and Actinobacteria were dominant bacteria in Y1.1, which accounted for $61.56 \%$ and $18.26 \%$ of the total bacteria. Proteobacteria, Acidobacteria and Actinobacteria were dominant bacteria in Y6.1, which accounted for $47.42 \%, 19.71 \%$ and $13.40 \%$ of the total bacteria. Proteobacteria, Bacteroidetes and Actinobacteria were dominant bacteria of Y7.1, which accounted for $54.28 \%, 15.59 \%$ and $11.24 \%$ of the total bacteria. Proteobacteria were the most dominant bacteria in all the 3 samples with proportions between $47.42 \%$ and $61.56 \%$. The sub dominant bacteria of 3 samples were different; in Y1.1 samples the sub dominant bacteria were Actinobacteria; in Y6.1 the sub dominant bacteria were Acidobacteria; in Y7.1 sample the sub dominant bacteria were Bacteroidetes.

To further understand the distribution and composition of endophytic bacteria in 3 tobacco samples, 10 OTUs with the highest abundance of bacteria in each sample were selected. Because the plant chloroplast 16S rDNA and mitochondrial 18S rDNA are highly homologous to the sequence of bacterial 16S rDNA, the 3 samples were all contaminated by Unidentified-Chloropiast and Unidentified-Mitochondria. The Y1.1 sample was contaminated by Unidentified-Chloropiast (66.16\%) and Unidentified-Mito. Chondria (13.20\%); Y6.1 sample was contaminated by Unidentified-Chloropiast (69.20\%) and Unidentified-Mitochondria (6.19\%); Y7.1 sample was contaminated by Unidentified-Chloropiast (42.48\%) and Unidentified-Mitochondria (4.35\%). After excluding host pollution, Y1.1 was Sphingomonas (0.86\%), Variovorax (0.05\%), Blastococcus (0.51\%), Lysobacter $(0.10 \%)$ and others $(19.10 \%)$; Y6.1 was Sphingomonas $(1.30 \%)$, Variovorax (0.07\%), Morganella (0.01\%), Blastococcus (0.30\%), Lysobacter (0.24\%) and others (22.70\%); Y7.1 was Sphingomonas (1.34\%), Variovorax (1.00\%), Fusobacterium (0.89\%), Morganella (0.59\%), Blastococcus (0.55\%), Proteiniclasticum (0.55\%), Lysobacter (0.54\%) and others (47.02\%). Sphingomonas, Variovorax, Blastococcus and Lysobacter could be found in all the three samples; Morganella could be found in Y6.1 and Y7.1; Fusobacterium and Proteiniclasticum could only be found in Y7.1. It can be seen from the experimental results that the distribution of Morganella endophytic bacteria in Y6.1 and Y7.1 was related to the addition of biological carbon. The distribution of Fusobacterium and Proteiniclasticum endophytic bacteria in Y7.1 was related to the inter cropping with marigold. 


\section{Conclusion and Discussion}

Studying the diversity of endophytic bacteria can help us to understand the interaction mechanism of endophytic bacteria better and utilize endophytic bacteria resources more effectively. In this experiment, Yunyan 87 was taken as the research material; high throughput sequencing technology which overcame the defects of low flux in traditional molecular biological method was employed. Compared with the traditional pure culture method and 16S rDNA non culture method, more comprehensive information about plant endophytic diversity was obtained. [10] In order to understand the distribution and composition of endophytic bacteria in 3 samples, 10 OTUs with the highest abundance of bacteria in each sample were compared. The study found that endophytic bacteria of the Morganella genus were distributed only in Y6.1 and Y7.1. Since $150 \mathrm{~g}$ biological carbon was applied to each plant of tobacco of Y6.1 and Y7.1, it is assumed that the distribution of Morganella endophytic bacteria in tobacco leaves is related to the addition of biological carbon. Fusobacterium and Proteiniclasticum endophytic bacteria were only distributed in Y7.1. Y1.1 and Y6.1 were single cropping tobacco; Y7.1 was tobacco inter cropping with marigold. Therefore, it is speculated that the distribution of Fusobacterium and Proteiniclasticum endophytic bacteria is related to the inter cropping with marigold. It can be seen that changes in planting pattern has great influence on the diversity of endophytic bacteria, which is in line with the research of Tong-suo Ma and his colleagues. [5] In further study, the relationship between Morganella, Fusobacterium and Proteiniclasticum endophytic bacteria and the growth as well as the stress resistance of tobacco plants could be analyzed. The effects of different planting methods on the cultivation of tobacco can be analyzed from the microbiological point of view, so as to provide more reliable technical reference for the adjustment of tobacco planting methods.

\section{Acknowledgements}

This paper is supported by National Natural Science Foundation of China (41361056, 31460491); Joint Special Fundamental Research of Local Universities in Yunnan Province (2017FH001-041); Talent Introduction Program of Kunming University (YJL14005); Key Disciplines (Ecology) Project of Yunnan Education Department (05000511311); Open Fund Project of Key Laboratory of Characteristic Biological Resources Development and Utilization in Yunnan Province (GXKZ201716).

\section{References}

[1] X.Z. Liu, Z.L. Zhao, S.B. Li, et al., The community structure and diversity of the endophytes in American ginseng, J. Acta microbiology Sinica. 55 (2015).

[2] H.J. Wan, Y.F. Xie, B. Xie, et al., Analysis the differences of endophyte bacterias in panxiginsengs by 16S rDNA-RFLP method, J. Journal of Jiangxi College of Traditional Chinese Medicine. 27 (2015).

[3] L.A. Yao, Z.B. Hu, L.L. Wang, et al., Research development of the relationship between plant endophyte and host, J. Ecology and Environment.19 (2010).

[4] T. Hashiba, K. Narisawa, The development and endophytic nature of the fungus Heteroconium chaetospira, J. FEMS Microbiology Letters. 252 (2005).

[5] T.S. Ma, M.Z. Tian, H.L. Yuan, et al., Effects of different growth environmental conditions on the distribution of endophytes in two species of vegetables, J. Journal of Anhui Agricultural Sciences. 37 (2009).

[6] X.Y. Liu, G.S. Liu, H.E. Liu, et al., Effects of biochar application amount on growth, yield and quality of tobacco, J. Journal of Henan Agricultural Sciences. 43 (2014).

[7] Z.B. Chen, F.P. Dai, L.J. Cun, et al., Research on separation optimization of endophytic bacteria in tobacco, J. Acta Tabacaria Sinica. 20 (2014). 
[8] F.B. Qiu, Exploration on Endophytic Bacteria in Panax Ginseng Roots with Culture-dependent and Culture-independent Approaches, Capital Normal University, Beijing, 2007.

[9] S. Jin, Z.B. Chen, Y.C. Li, et al., Endophytic bacterial diversity of peperomia dindygulensis miq. estimated via high throughput sequencing, J. Chinese Journal of Tropical Crops.38 (2017).

[10] J. Li, F.S. Liu, Q. Gao, et al., Effects of application of different bio-organic fertilizer with tobacco specific chemical fertilizer on the amount of microbe and enzyme activities in the rhizosphere soil of flue-cured tobacco, J. Journal of Henan Agricultural University. 47 (2013). 